\title{
Clinicopathological features and prognostic analysis of 49 cases with crescentic glomerulonephritis
}

\author{
TING WU ${ }^{1 *}$, JIAJIA PENG ${ }^{1 *}$, TING MENG ${ }^{1}$, QIANQIAN LIU ${ }^{1}$, XIANG AO $^{1}$, WEI LIN ${ }^{2}$, \\ HONGLING YIN ${ }^{2}$, JINBIAO CHEN ${ }^{3}$, JIAXI PU ${ }^{1}$, ZHANGZHE PENG ${ }^{1}$, WEISHENG PENG ${ }^{1}$, \\ XIAOZHAO LI $^{1}$, XIANGCHENG XIAO ${ }^{1}$, QIAOLING ZHOU ${ }^{1}$, YONG ZHONG ${ }^{1}$ and PING XIAO ${ }^{1}$ \\ Departments of ${ }^{1}$ Nephrology, ${ }^{2}$ Pathology and ${ }^{3}$ Medical Records and Information, \\ Xiangya Hospital, Central South University, Changsha, Hunan 410008, P.R. China
}

Received January 14, 2019; Accepted July 16, 2019

DOI: $10.3892 /$ etm.2019.8023

\begin{abstract}
Rapidly progressive glomerulonephritis (RPGN), characterized by rapid kidney dysfunction caused by aggressive glomerulonephritis, is usually associated with crescentic glomerulonephritis $(\mathrm{CrGN})$. In the present study, the data from patients with $\mathrm{CrGN}$ were retrospectively analyzed at a tertiary medical center in China with the aim of investigating the clinicopathological features and the association of the type of CrGN with the prognosis. The renal biopsies of 49 patients diagnosed with CrGN were obtained between December 2011 and July 2016. Of the 49 patients, 11 patients $(22.45 \%)$ had type I CrGN, 19 (38.78\%) had type II CrGN and 19 (38.78\%) had type III CrGN. The majority of CrGN patients exhibited multiple-system involvement and 28 patients $(57.14 \%)$ had kidney enlargement. Proportions of patients with acute kidney injury (AKI), acute kidney diseases without AKI, and chronic kidney disease were $28.57,46.94$ and $24.49 \%$, respectively. Among the 3 types of $\mathrm{CrGN}$, patients with type I CrGN tended to have a higher proportion of AKI with more cellular crescent formation, and higher serum creatinine and retinol binding protein. Circulating anti-GBM antibodies were present in all type I CrGN patients and anti-neutrophilic cytoplasmic autoantibodies were detected in $84.21 \%$ of patients with type III CrGN. Type III CrGN patients had a superior kidney survival, whereas type I CrGN patients had the worst kidney prognosis $(\mathrm{P}<0.001)$. There was no significant difference in overall patient survival among the 3 types of CrGN. CrGN remains the primary cause of critical illness in RPGN patients.
\end{abstract}

Correspondence to: Dr Yong Zhong or Professor Ping Xiao, Department of Nephrology, Xiangya Hospital, Central South University, 87 Xiangya Road, Changsha, Hunan 410008, P.R. China E-mail: zhongyong121@163.com

E-mail: xiaoping.x@163.com

*Contributed equally

Key words: crescentic glomerulonephritis, clinical pathology, treatment and prognosis
There was much heterogeneity between the different subtypes of CrGN. Patients with type I tended to have an acute onset and had the poorest kidney survival.

\section{Introduction}

Rapidly progressive glomerulonephritis (RPGN) has been characterized by the rapid loss of kidney function and is usually caused by crescentic glomerulonephritis ( $\mathrm{CrGN})$ within a few weeks or months. CrGN is defined as crescents involving $>50 \%$ of the glomeruli (1). CrGN can be divided into 3 types according to immunofluorescence microscopy: Type I is defined as a linear deposition of immunoglobulins along the glomerular basement membrane (GBM); type II is defined as glomerular immune complex deposition; and type III is defined as glomerular pauci-immune deposition (2). Epidemiologic data on $\mathrm{CrGN}$ have been reported by large national kidney biopsy registries from India (3), Japan (4), Saudi Arabia (5), the US (6), Spain (7) and China (8,9). The prevalence of CrGN ranges from 1.56 to $10 \%$ of total kidney biopsies.

The exact mechanism of immune-pathogenesis in crescent formation remains elusive. Studies have revealed that a key stage is the breakage of the GBM, allowing plasma proteins to enter the Bowman's capsule (10-12). A second key stage in crescent formation is the accumulation of fibrin within the Bowman's capsule, which provokes the proliferation of parietal epithelial cells. In addition, macrophages, $\mathrm{CD}^{+}{ }^{+} \mathrm{T}$ cells and $\mathrm{CD}^{+} \mathrm{T}$ cells are important molecules that are involved in the immune-pathogenesis of crescent formation $(10,13)$. The standard induction therapy for $\mathrm{CrGN}$ includes oral prednisone combined with cyclophosphamide (CTX) (10). Many physicians also frequently use pulse methylprednisolone prior to the administration of high dose oral steroids in addition to CTX. Plasmapheresis may be also used to remove circulating auto-antibodies or immune complexes. Early treatment is of vital importance for patients with CrGN (14). The prognosis of patients with $\mathrm{CrGN}$ is regarded as having been improved over the past few years. The 5-year cumulative renal survival rates of patients with type I, II and III CrGN have reached 17.6, 70.1 and 44.3\%, respectively, in China (8). Certain clinicopathological features, including the subtype (8), oliguria and serum creatinine ( $\mathrm{SCr}$ ) (3), age and an elevated percentage of 
glomeruli with crescents (5), have a significant impact on the prognosis of patients with CrGN. The purpose of the present study was to assess the clinicopathological features and outcomes of patients with CrGN.

\section{Patients and methods}

Population and data collection. A total of 49 consecutive patients with biopsy-proven $\mathrm{CrGN}$ diagnosed between December 2011 and July 2016 at the Department of Nephrology of Xiangya Hospital of Central South University (Changsha, China) were recruited for the present retrospective study. During this period, 1,312 renal biopsies were performed. Patients with $<10$ non-sclerotic glomeruli in sampled renal tissue were excluded. CrGN was defined as the presence of crescents within the Bowman's space in $>50 \%$ of the total glomeruli in the kidney biopsy (15).

Once patients provided written informed consent to participate in the study, baseline demographic data, including age, sex, duration of disease and clinicopathological, laboratory and ultrasonography parameters, were obtained from the electronic medical record system of the hospital. Details regarding the process of treatment and outcome, including SCr levels, dependence on dialysis, outcome concerning survival and cause of death, were also collected. Patients were evaluated at the time of diagnosis, at 1,2 and 3 months, and then every 3 months until the end of the study. Patients were followed up from diagnosis until end-stage renal disease (ESRD) was reached or death or the final follow-up date (July 30, 2017). All follow-up data were collected from the hospital's electronic medical records and by contacting the individual patients directly.

Classification of $C r G N$. As described previously, each renal specimen was assessed by direct immunofluorescence, light and electron microscopy (16). These experiments had been performed with the samples at the time of diagnosis. CrGN was defined as $\geq 50 \%$ of the total glomeruli having large crescents as evaluated by light microscopy. On the basis of the immunofluorescence microscopy results, $\mathrm{CrGN}$ was classified into 3 types. By definition, type I CrGN exhibited linear deposits of immunoglobulins (Ig) along the GBM, which was always accompanied by anti-GBM antibodies in the serum. Type II was characterized by granular immune-complex deposition on the glomerular tuft, while in type III, which is also referred to as pauci-immune $\mathrm{CrGN}$, immunofluorescence was negative or deposits of Ig were rare (2).

Definitions. The definitions of acute kidney injury (AKI) and chronic kidney disease (CKD) are widely accepted, while acute kidney diseases and disorders (AKD) is a relatively novel concept (17). Hence, the criteria for AKI, AKD without AKI and CKD were used to illustrate the different clinical characteristics of patients with CrGN. AKI was defined as an increase in $\mathrm{SCr}$ by $\geq 0.3 \mathrm{mg} / \mathrm{dl}(26.5 \mu \mathrm{mol} / \mathrm{l})$ within $48 \mathrm{~h}$ or an increase in $\mathrm{SCr}$ to 1.5 times the baseline value within 7 days or a urine volume of $<0.5 \mathrm{ml} / \mathrm{kg} / \mathrm{h}$ over $6 \mathrm{~h}$. AKD without AKI was defined as an estimated glomerular filtration rate (eGFR) of $<60 \mathrm{ml} / \mathrm{min}$ per $1.73 \mathrm{~m}^{2}$ for $<3$ months, a decrease in eGFR $\geq 35 \%$, an increase in $\mathrm{SCr}>50 \%$ or abnormalities of kidney structure based on urinary markers and imaging studies for $<3$ months. CKD was defined as eGFR $<60 \mathrm{ml} / \mathrm{min}$ per $1.73 \mathrm{~m}^{2}$ or kidney structural damage for $>3$ months (8).

In addition to the major features of the kidney, certain extrarenal manifestations coexisted, including hematological disorders, serositis and pneumonia. For simplicity, multiple-system involvement (MSI) was uniformly defined to summarize the clinical manifestation, i.e. involvement of at least one other organ in addition to the kidney. It is worth mentioning that, since anemia in $\mathrm{CrGN}$ is one of the most common complications, it was not considered to be an organ-involving manifestation.

Statistical analysis. All data were analyzed using SPSS version 19.0 (IBM Corp.). Quantitative data were expressed as the mean \pm standard deviation, $\mathrm{n}(\%)$ or median with interquartile range. All parameters were compared using the $\chi^{2}$ test or Fisher's exact test for categorical data and one-way analysis of variance (ANOVA) or Kruskal-Wallis test for continuous data. The least-significant difference test and Bonferroni correction were used as post-hoc tests that followed ANOVA and the Kruskal-Wallis test respectively. Kaplan-Meier curves with log-rank tests were used to analyze patient survival as well as renal survival. $\mathrm{P}<0.05$ was considered to indicate statistical significance.

\section{Results}

Demographics and kidney manifestations. In the present retrospective study, CrGN accounted for 3.73\% (49/1312) of the total patients receiving renal biopsies during the study period at the Department of Nephrology, Xiangya Hospital of Central South University (Changsha, China). Of the 49 cases identified, $11(22.45 \%)$ patients were classified as type I, 19 (38.78\%) as type II and the remaining 19 (38.78\%) as type III. A total of $23(46.94 \%)$ were females and $26(53.06 \%)$ were males, with an average age of $44.51 \pm 15.95$ years at the time-point of diagnosis (Table I). The duration of symptoms prior to admission varied greatly from 7 days to 13 months, with a median duration of 86 days. However, no significant differences in the demographic data were determined among the 3 types of $\mathrm{CrGN}$.

Concerning the clinical characteristics, MSI was observed in most CrGN patients (97.96\%), 29 patients $(59.18 \%)$ had hypertension and 9 (18.37\%) had oliguria. Furthermore, all CrGN patients had hematuria, including microscopic or gross hematuria. The proportions of patients with AKI, AKD without AKI, and CKD were 28.57, 46.94 and 24.49\%, respectively. In addition, patients with type I disease tended to have an acute onset, with $72.73 \%$ of patients presenting with AKI, which was significantly different from the other two groups ( $\mathrm{P}=0.001$; Table I).

Laboratory and ultrasonography data. In general, patients with kidney diseases have varying degrees of anemia (15), and this was also observed in the CrGN patients of the present study. As presented in Table II, patients with type II CrGN had less severe anemia than the other two subtypes $(\mathrm{P}<0.05$ type II vs. type I; $\mathrm{P}<0.05$ type II vs. type III). The average baseline $\mathrm{SCr}$ level of all patients was $612 \pm 416 \mu \mathrm{mol} / \mathrm{l}$. In 
Table I. Baseline demographic and clinicopathological characteristics of patients with crescentic glomerulonephritis.

\begin{tabular}{|c|c|c|c|c|c|}
\hline Item & Total $(n=49)$ & Type I $(\mathrm{n}=11)$ & Type II $(\mathrm{n}=19)$ & Type III (n=19) & P-value \\
\hline Age (years) & $44.51 \pm 15.95$ & $37.09 \pm 15.14$ & $43.74 \pm 18.06$ & $49.58 \pm 12.80$ & 0.113 \\
\hline Female sex & $23(46.94)$ & $3(27.27)$ & $12(63.16)$ & $8(42.11)$ & 0.143 \\
\hline Duration of disease (days) & $86(30-100)$ & $52(17.5-60)$ & $90(25.5-90)$ & $102(32.5-120)$ & 0.16 \\
\hline Hypertension & $29(59.18)$ & $8(72.73)$ & $10(52.63)$ & $11(57.89)$ & 0.531 \\
\hline Oliguria & $9(18.37)$ & $3(27.27)$ & $2(10.53)$ & $4(21.05)$ & 0.484 \\
\hline Anuria & $3(6.12)$ & $1(9.09)$ & $1(5.26)$ & $1(5.26)$ & 0.905 \\
\hline AKI & $14(28.57)$ & $8(72.73)$ & $3(15.79)^{\mathrm{a}}$ & $3(15.79)^{\mathrm{a}}$ & 0.001 \\
\hline AKD without AKI & $23(46.94)$ & $2(18.18)$ & $11(57.89)$ & $10(52.63)$ & 0.09 \\
\hline CKD & $12(24.49)$ & $1(9.09)$ & $5(26.32)$ & $6(31.58)$ & 0.404 \\
\hline MSI & $48(97.96)$ & $10(90.91)$ & $19(100)$ & $19(100)$ & 0.224 \\
\hline
\end{tabular}

Values for categorical variables are presented as $\mathrm{n}(\%)$ and values for continuous variables as the mean \pm standard deviation or median (interquartile range). ${ }^{\mathrm{a}} \mathrm{P}<0.05$ vs. type I. AKD, acute kidney diseases and disorders; AKI, acute kidney injury; CKD, chronic kidney disease; MSI, multiple-system involvement.

Table II. Laboratory and ultrasonography data by type of crescentic glomerulonephritis.

\begin{tabular}{lcccc}
\hline Parameter & Total $(\mathrm{n}=49)$ & Type I $(\mathrm{n}=11)$ & Type II $(\mathrm{n}=19)$ & Type III $(\mathrm{n}=19)$ \\
\hline Hemoglobin $(\mathrm{g} / \mathrm{l})$ & $83.98 \pm 20.87$ & $78.18 \pm 22.35$ & $94.74 \pm 19.98^{\mathrm{a}}$ & $76.58 \pm 16.87^{\mathrm{b}}$ \\
Albumin $(\mathrm{g} / \mathrm{l})$ & $28.1 \pm 5.05$ & $26.98 \pm 3.77$ & $28.15 \pm 5.61$ & $28.71 \pm 5.64$ \\
Proteinuria (g/day) & $2.32 \pm 2.49$ & $2.90 \pm 3.85$ & $2.91 \pm 2.43$ & $1.39 \pm 1.14^{\mathrm{a}, \mathrm{b}}$ \\
Scr $(\mu$ mol/l) & $612 \pm 416$ & $928 \pm 381$ & $502 \pm 410^{\mathrm{a}}$ & $537 \pm 365^{\mathrm{a}}$ \\
NAG (u/l) & $28.3 \pm 18.7$ & $23.9 \pm 11.8$ & $31.0 \pm 22.3$ & $26.9 \pm 17.0$ \\
RBP (mg/l) & $14.9 \pm 19.3$ & $36.6 \pm 27.0$ & $16.3 \pm 20.5^{\mathrm{a}}$ & $6.7 \pm 6.7^{\mathrm{a}}$ \\
C4 (mg/l) & $253(208-281)$ & $309(235-382)$ & $239(208-274)^{\mathrm{a}}$ & $233(194-264)^{\mathrm{a}}$ \\
C3 (mg/l) & $898(744-1,045)$ & $1,039(830-1,230)$ & $882(758-984)$ & $829(681-978)$ \\
IgG (g/l) & $12.7(7.9-16.5)$ & $9.2(7.0-10.0)$ & $10.8(6.7-14.3)$ & $17.0(13.9-20.2)^{\mathrm{a}, \mathrm{b}}$ \\
IgA (mg/l) & $2,611(1,675-3,285)$ & $2,193(1,385-2,430)$ & $2,678(1,790-3,255)$ & $2,807(1,770-3,610)$ \\
IgM (mg/l) & $1,232(811-1,540)$ & $1,124(723-1,490)$ & $1,298(843-1,710)$ & $1,228(860-1,530)$ \\
ANCA positive & $19 / 46(41.30)$ & $0 / 10(0)$ & $3 / 17(17.65)$ & $16 / 19(84.21)^{\mathrm{a}, \mathrm{b}}$ \\
GBM & $12 / 32(37.50)$ & $11 / 11(100)$ & $0 / 9(0)^{\mathrm{a}}$ & $1 / 12(8.33)^{\mathrm{a}}$ \\
MPO-ANCA positive & $13 / 32(40.63)$ & $0 / 11(0)$ & $2 / 9(22.22)$ & $1 / 12(91.67)^{\mathrm{a}, \mathrm{b}}$ \\
PR3-ANCA positive & $1 / 32(3.13)$ & $0 / 11(0)$ & $11.11)$ & $0 / 12(0)$ \\
Kidney enlargement & $28 / 49(57.14)$ & $8 / 11(72.73)$ & $8 / 19(42.11)$ \\
\hline
\end{tabular}

Values for categorical variables are given as $\mathrm{n}(\%)$ and values for continuous variables as the mean \pm standard deviation or median (interquartile range). ${ }^{\mathrm{a}} \mathrm{P}<0.05$ vs. type I and ${ }^{\mathrm{b}} \mathrm{P}<0.05$ vs. type II. Scr, serum creatinine; ANCA, antineutrophil cytoplasmic antibody; C3, complement 3; IgA, immunoglobulin A; GBM, glomerular basement membrane; MPO, myeloperoxidase; NAG, N-acetyl-b-D-glucosaminidase; PR3, proteinase 3; RBP, retinol binding protein.

accordance with the clinical presentation, the SCr level and the kidney tubular injury parameter retinol binding protein of patients with type I CrGN were markedly higher than those in patients with the other two types $(\mathrm{P}<0.05)$. Of the patients with type III CrGN, $84.21 \%$ had serum anti-neutrophilic cytoplasmic autoantibodies (ANCA), and they had a lower amount of proteinuria. As expected, circulating anti-GBM antibodies were detected in all patients with type I CrGN. In addition, patients with type I CrGN had a high level of serum complement 4 ( $\mathrm{P}<0.05$ type I vs. type II; $\mathrm{P}<0.05$ type I vs. type III) and patients with type III CrGN had a relatively high level of serum $\operatorname{IgG}(\mathrm{P}<0.05$ type III vs. type $\mathrm{I} ; \mathrm{P}<0.05$ type III vs. type II). Finally, kidney enlargement was identified in $57.14 \%$ of patients. However, there were no obvious differences in kidney enlargement or serum albumin levels among the different types of CrGN.

Primary diseases of patients with type II CrGN. Regarding primary disease, the composition of the 19 patients with type II CrGN was as follows: 8/19 (42.11\%) had $\operatorname{Ig} \mathrm{A}$ 
Table III. Primary diseases of patients with type II CrGN.

$\mathrm{n}(\%$ of

Variables

Type II CrGN)

IgA nephropathy, n (\%)

$8 / 19(42.11)$

Lupus nephritis, n (\%)

$4 / 19(21.05)$

Anti-nuclear antibodies positive, n (\%)

$4 / 4(100)$

$3 / 4(75)$

Anti-double stranded DNA antibodies

Anti-Smith antibodies positive, $\mathrm{n}(\%)$

$3 / 19(15.79)$

ANCA-associated glomerulonephritis, n (\%)

$2 / 19(10.53)$

$\mathrm{H}-\mathrm{S}$ purpura glomerulonephritis, n (\%)

$1 / 19(5.26)$

Hepatitis B virus-associated nephritis, n (\%)

$1 / 19(5.26)$

CrGN,crescentic glomerulonephritis;IgA,immunoglobulin A;ANCA, anti-neutrophilic cytoplasmic autoantibodies; H-S,Henoch-Schönlein.

nephropathy, 4/19 (21.05\%) had lupus nephritis, 3/19 (15.79\%) had ANCA-associated glomerulonephritis, 2/19 (10.53\%) had Henoch-Schönlein purpura glomerulonephritis, 1/19 $(5.26 \%)$ had hepatitis B virus-associated nephritis and 1/19 (5.26\%) had idiopathic immune-complex CrGN (Table III). All 4 patients diagnosed with lupus nephritis had serum anti-nuclear antibodies, 3 of whom had anti-double stranded DNA (anti-dsDNA) antibodies and 1 had positive anti-Smith antibodies.

Pathological characteristics. Renal biopsy from a representative case of type I CrGN revealed predominant cellular crescents and linear deposits of IgG along the GBM on immunofluorescence (Fig. 1). Among all of the subjects with CrGN, the mean overall percentages of glomeruli with crescents, cellular crescents and fibrous crescents were $65.84 \pm 13.25$, $35.34 \pm 17.81$ and $30.49 \pm 15.71 \%$, respectively (Table IV). Compared with the other two subtypes, patients with type I CrGN had a significantly higher percentage of cellular crescents $(\mathrm{P}<0.05)$. Furthermore, patients with type II CrGN had a lower percentage of sclerosis than type III.

Treatment, and overall and renal survival. As presented in Table V, only 14 patients $(28.57 \%)$ of the present cohort received plasma exchange, and even of the patients with type I CrGN, only $63.64 \%$ received plasma exchange. With regard to the treatment of plasma exchange between type I CrGN and type II CrGN, the difference was significant $(\mathrm{P}<0.05)$. Of the total patients, $48(97.96 \%)$ and $41(83.67 \%)$ received corticosteroids and cyclophosphamide, respectively. A total of 35 patients $(71.43 \%)$ were treated with intravenous pulses of methylprednisolone at diagnosis.

The renal survival of the patients is presented in Fig. 2. Patients with type III CrGN had superior renal survival, whereas those with type I CrGN had the poorest renal prognosis ( $\mathrm{P}=0.002$, type I vs. type II; $\mathrm{P}<0.01$, type I vs. type III; $\mathrm{P}=0.15$, type II vs. type III). Regarding patient survival, no significant difference was present among the 3 types of $\mathrm{CrGN}$ (Fig. 3).

\section{Discussion}

Validation studies of epidemiologic data on $\mathrm{CrGN}$ have been performed in certain countries (4). In the present retrospective study, $\mathrm{CrGN}$ accounted for $3.73 \%$ of the total patients receiving renal biopsies during the study period at our center, which is higher than previously reported rates from China $(8,9,15)$, but was similar to results from an Indian study (3). In addition, an equal proportion of pauci-immune and immune complex GN was encountered in patients with $\mathrm{CrGN}$ at our center, followed by anti-GBM disease. This result was different from that of several previous studies, by which pauci-immune CrGN was reported to be the predominant type $(3,4,6,7)$. In Europe, the annual incidence of renal vasculitis was reported to be 10-20 per 1 million individuals (18); the incidence in China is currently not available. According to a study by Chen et al (19) $>400$ patients with ANCA-associated vasculitis (AAV) were diagnosed during an 8-year period in their referral diagnostic center in Peking University First Hospital in China. Anti-GBM disease is considered a rare disease, with an incidence of 1-2 cases per million per year in China (20). While the incidence in China is not available, $>30$ anti-GBM-positive sera from new patients are screened annually at Peking University First Hospital (21), indicating that, relative to other hospitals worldwide, hospitals in China encounter more patients with anti-GBM disease. The present study reported a higher proportion of patients with type I CrGN among the CrGN cases studied compared with the results of previous studies $(3,4,6,7)$ and further supports the notion that hospitals in China may encounter more patients with anti-GBM disease. In type II CrGN, IgA nephropathy was the most common primary disease, which is consistent with previous studies (16,21-25).

In certain severely ill patients, renal biopsy cannot always be smoothly and quickly performed. Thus, specific serum markers [serum myeloperoxidase (MPO-ANCA) or proteinase 3 (PR3-ANCA)] are important and are correlated with AAV. Of those patients with granulomatosis with polyangiitis (Wegener's granulomatosis), 90\% have PR3-ANCA and patients with microscopic polyangiitis have the highest frequency of MPO-ANCA (26). ANCA themselves are deemed to be pathogenic in these diseases (27). Serum ANCA is considered a basis for the recognition of ANCA-associated vasculitis, which frequently presents as type III CrGN. However, it was also detected in a small number of patients with type I and II CrGN. It was reported that patients with negative ANCA had better renal outcomes $(8,28,29)$. However, others have reported that patients without ANCA had higher levels of proteinuria, more severe glomerular lesions and poorer renal outcomes (8). In the present study, the majority of patients with type III CrGN were MPO-ANCA-positive, which was similar to the result of a previous study from China (8). In the present cohort, serum ANCA was detected in $17.65 \%$ of cases with type II CrGN, which is higher than the percentage reported in the above previous study (8). Lin et al (15) reported that glomerular necrosis and crescent formation were associated with ANCA in immune-complex CrGN. Furthermore, ANCA and immune complex deposition possibly act synergistically (15). The renal survival in patients with type II CrGN in the present study was lower than that previously reported $(3,8,9)$, perhaps due to a higher ratio of serum ANCA in type II CrGN. 
Table IV. Pathological characteristics of patients with crescentic glomerulonephritis.

\begin{tabular}{lllll}
\hline Item & Total $(\mathrm{n}=49)$ & Type I $(\mathrm{n}=11)$ & Type II $(\mathrm{n}=19)$ & Type III $(\mathrm{n}=19)$ \\
\hline Cellular crescents $(\%)$ & $35.34 \pm 17.81$ & $48.31 \pm 18.69$ & $32.67 \pm 16.61^{\mathrm{a}}$ & $30.52 \pm 15.51^{\mathrm{a}}$ \\
Fibrous crescents (\%) & $30.49 \pm 15.71$ & $22.46 \pm 13.18$ & $31.58 \pm 16.65$ & $34.06 \pm 15.18$ \\
Sclerosis (\%) & $19.45 \pm 18.16$ & $21.53 \pm 25.48$ & $12.63 \pm 13.84$ & $25.05 \pm 15.56^{\mathrm{b}}$ \\
Crescents $(\%)$ & $65.84 \pm 13.25$ & $70.77 \pm 14.67$ & $64.24 \pm 13.22$ & $64.58 \pm 12.43$ \\
\hline
\end{tabular}

Values are expressed as the mean \pm standard deviation. ${ }^{\mathrm{a}} \mathrm{P}<0.05$ vs. type $\mathrm{I},{ }^{\mathrm{b}} \mathrm{P}<0.05$ vs. type II.

Table V. Treatment of patients with crescentic glomerulonephritis.

\begin{tabular}{|c|c|c|c|c|c|}
\hline Item & Total $(n=49)$ & Type I $(n=11)$ & Type II (n=19) & Type III $(n=19)$ & P-value \\
\hline Plasma exchange & $14(28.57)$ & $7(63.64)$ & $1(5.26)^{\mathrm{a}}$ & $6(31.58)$ & 0.003 \\
\hline Cyclophosphamide & $41(83.67)$ & $10(90.91)$ & $15(78.95)$ & $16(84.21)$ & 0.885 \\
\hline Corticosteroids & $48(97.96)$ & $11(100)$ & $19(100)$ & $18(94.74)$ & 1.000 \\
\hline Methylprednisolone pulse & $35(71.43)$ & $10(90.91)$ & $15(78.95)$ & $10(52.63)$ & 0.056 \\
\hline Other immunosuppressive agents & $9(18.37)$ & $2(18.18)$ & $5(26.32)$ & $2(10.53)$ & 0.522 \\
\hline
\end{tabular}

Values are expressed as $\mathrm{n}(\%) ;{ }^{\mathrm{a}} \mathrm{P}<0.05$ vs. type I.
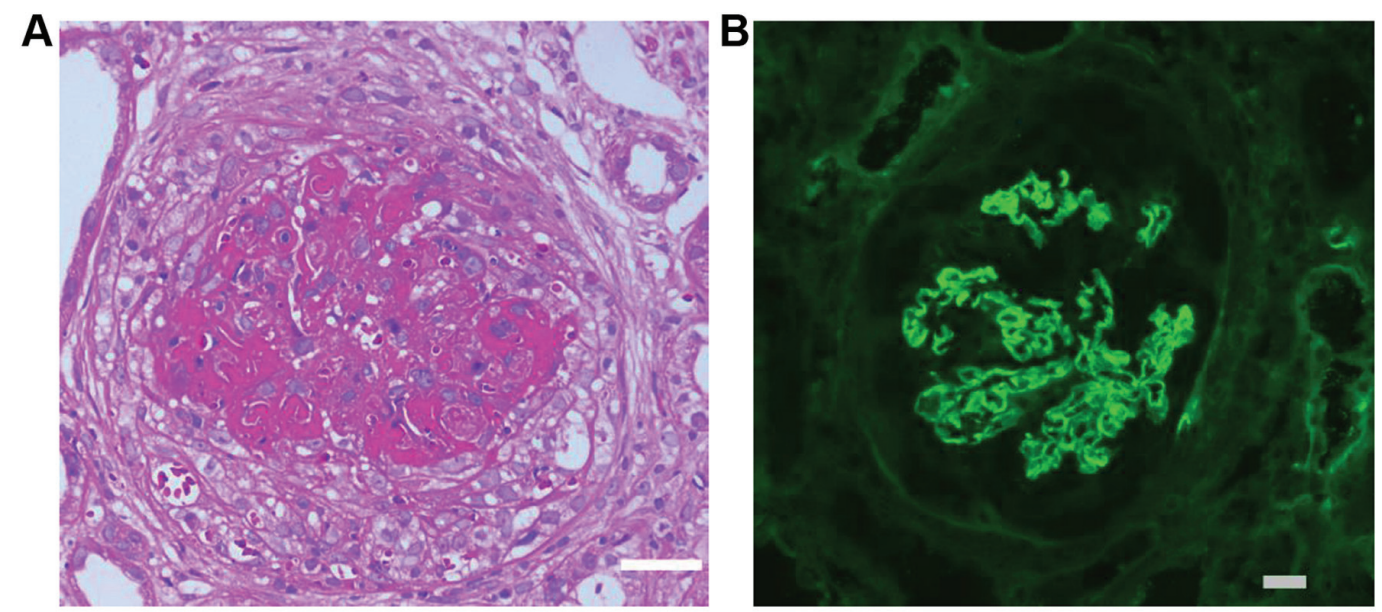

Figure 1. (A) Renal biopsy from a case of type I crescentic glomerulonephritis exhibiting predominantly cellular crescents (H\&E staining; scale bar, $40 \mu \mathrm{m})$. (B) Linear deposits of IgG along the glomerular basement membrane (green) on immunofluorescence (scale bar, $40 \mu \mathrm{m}$ ).

Several clinical characteristics were reported to be associated with renal outcome in $\mathrm{CrGN}$, including oliguria and $\mathrm{SCr}(3,8)$. A clinical grading system for patients with RPGN based on SCr and other clinical manifestations has been established to predict the prognosis (4). The average SCr concentration of patients at our center was much higher than that of patients from Spain (7), India (3), the US (6) and Japan (4). Patients with pauci-immune $\mathrm{CrGN}$ had higher $\mathrm{SCr}$ levels than those with immune complex $\mathrm{CrGN}$ at presentation in certain studies from the US (6) and Saudi Arabia (5), which was consistent with the present results. The medium proteinuria did not reach the standard of nephrotic syndrome, which was lower than that in studies from Saudi Arabia (5) and the US (6). Oliguria was similar to another study from China (8).
The association between histopathological features and prognosis has been previously reported $(3,5)$. The percentage of sclerosed glomeruli is one of the independent predictors of renal death $(5,8)$. The percentage of sclerosed glomeruli was determined to be significantly different between the pauci-immune and immune complex $\mathrm{CrGN}$, but renal survival was comparable in the two groups in the presnt study. The average percentage of sclerosed glomeruli in the current study was lower than that in another study from Saudi Arabia (5). There were no significant differences in the proportions of glomeruli exhibiting crescents and fibrous crescents between the three groups in the current study, which was consistent with the results of previous studies $(3,5,8)$. 


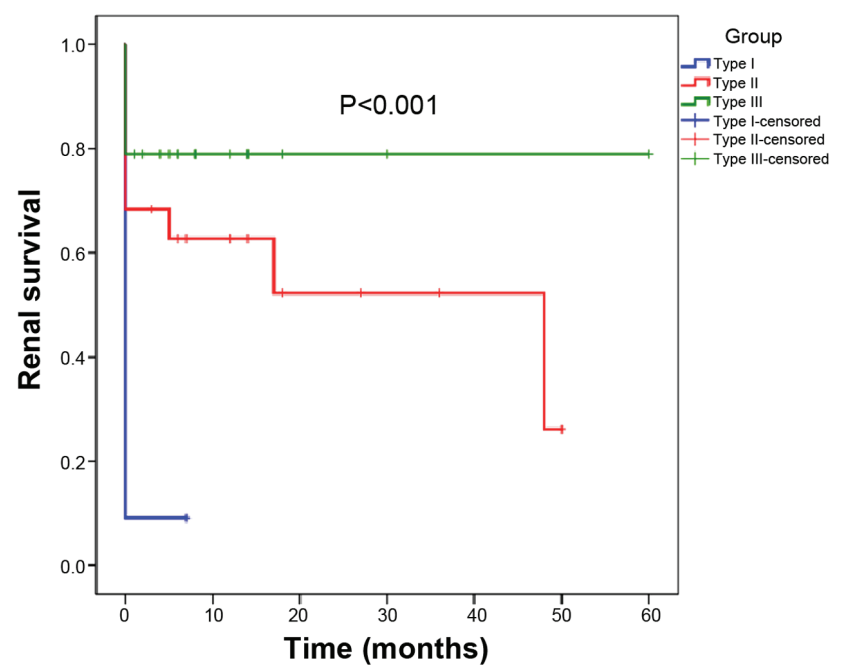

Figure 2. Renal survival of patients with different types of crescentic glomerulonephritis. Time (months) refers to the time since the start of treatment. $\mathrm{P}=0.002$, type I vs. type II; $\mathrm{P}<0.01$, type I vs. type III; $\mathrm{P}=0.15$, type II vs. type III.

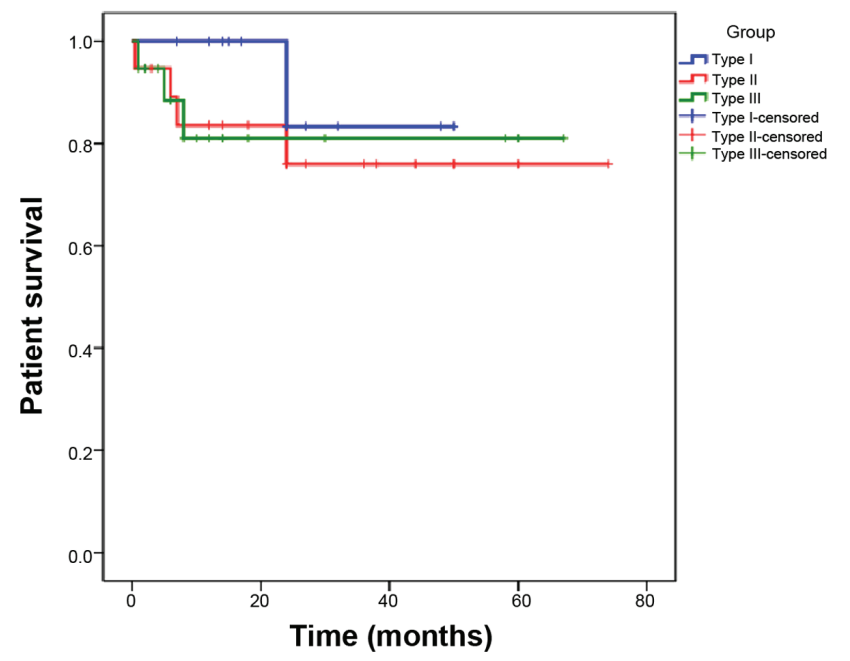

Figure 3. Survival of patients with different types of crescentic glomerulonephritis. Time (months) refers to the time since the start of treatment.

Renal replacement therapy in ESRD patients has a high cost (30). In the present study, patients with type I CrGN had the poorest renal prognosis, which has also been confirmed by previous studies $(3,6,8)$. The 1 -year kidney survival rate in the present study was lower than that reported previously $(31,32)$. This discrepancy in results may be explained by the higher initial average SCr concentration in the present study compared to those in the previous studies $(8,31)$. The low proportion of patients who received plasma exchange and less intensive immunosuppressive therapy may be another important reason.

Determining which type of CrGN between pauci-immune CrGN and immune complex CrGN is associated with better renal survival remains elusive. Several studies have indicated that the renal prognosis of patients with type II CrGN was superior to that of patients with type III CrGN $(3,8)$, which is in contrast to the results reported by Han et al (33). Of note, in the present study, renal survival in patients with type III CrGN was better than that in patients with type II CrGN, although the difference did not reach statistical significance. This comparison warrants further investigation in larger studies in the future.

The present study has several limitations. First, due to inclusion of only a small number of patients in the present study, there may have been a selection bias. Furthermore, follow-up was limited and a longer follow-up may be required in future studies. However, with the extension of the follow-up period, the number of cases lost to follow-up is expected to increase.

In conclusion, the present retrospective study reported that CrGN occurred in $3.37 \%$ of the total patients receiving renal biopsies during the study period at our center, which is higher than the rates reported by previous studies from China $(8,15)$. Type I CrGN had the poorest renal prognosis. The 1-year kidney survival rate in the present study was much lower than those reported by previous studies $(31,32)$. The results of the present study highlight the requirement for better treatments for this disease. A large national investigation on $\mathrm{CrGN}$ is required to improve the clinical management of these patients in the near future.

\section{Acknowledgements}

Parts of the present study were presented at the ISN World Congress of Nephrology, April 12-15, 2019 in Melbourne, Australia.

\section{Funding}

The present study was supported by the National Natural Science Foundation of China (grant nos. 81402453 and 81800641), the Natural Science Foundation of Hunan Province (grant no. 2015JJ4058), the Project of Hunan Health Commission of Hunan province (grant no. C2019184) and the Project of Hunan Development and Reform Commission [grant no. (2013) 1199], China.

\section{Availability of data and materials}

The datasets used and/or analyzed during the current study are available from the corresponding author on reasonable request.

\section{Authors' contributions}

YZ, JBC, TW and JJP designed the study, analyzed the data and wrote the manuscript. TM, QQL, XA, HLY, JXP, ZZP, WSP, XZL, XCX, QLZ and PX contributed to patient enrollment and follow-up. WL and HLY analyzed the pathological data. YZ and JBC analyzed the data. All authors read and approved the final manuscript.

\section{Ethics approval and consent to participate}

The present study was approved by the ethics review committee of Xiangya Hospital Central South University (reference no. 201403061). All patients provided written informed consent to participate in the study. 


\section{Patient consent for publication}

Not applicable.

\section{Competing interests}

The authors declare that they have no competing interests.

\section{References}

1. Pettersson EE, Sundelin B and Heigl Z: Incidence and outcome of pauci-immune necrotizing and crescentic glomerulonephritis in adults. Clin Nephrol 43: 141-149, 1995.

2. Couser WG: Rapidly progressive glomerulonephritis: Classification, pathogenetic mechanisms, and therapy. Am J Kidney Dis 11: 449-464, 1988

3. Gupta R, Singh L, Sharma A, Bagga A, Agarwal SK and Dinda AK: Crescentic glomerulonephritis: A clinical and histomorphological analysis of 46 cases. Indian J Pathol Microbiol 54 497-500, 2011.

4. Koyama A, Yamagata K, Makino H, Arimura Y, Wada T, Nitta K, Nihei H, Muso E, Taguma Y, Shigematsu H, et al: A nationwide survey of rapidly progressive glomerulonephritis in Japan: Etiology, prognosis and treatment diversity. Clin Exp Nephrol 13: 633-650, 2009.

5. Oudah N, AlDuhailibZ, Alsaad K, Qurashi S, Ghamdi G,Flaiw A Hejaili F, Farooqui M and Al Sayyari A: Glomerulonephritis with crescents among adult Saudi patients outcome and its predictors. Clin Exp Med 12: 121-125, 2012.

6. Jennette JC: Rapidly progressive crescentic glomerulonephritis Kidney Int 63: 1164-1177, 2003.

7. Lopez-Gómez JM and Rivera F; Spanish Registry of Glomerulonephritis: Renal biopsy findings in acute renal failure in the cohort of patients in the Spanish Registry of Glomerulonephritis. Clin J Am Soc Nephrol 3: 674-681, 2008.

8. Chen S, Tang Z, Xiang H, Li X, Chen H, Zhang H, Hu W, Zeng C and Liu Z: Etiology and outcome of crescentic glomerulonephritis from a single center in china: A 10-year review. Am J Kidney Dis 67: 376-383, 2016.

9. Tang Z, Wu Y, Wang Q, Zeng C, Yao X, Hu W, Chen H, Liu Z and Li L: Clinical spectrum of diffuse crescentic glomerulonephritis in Chinese patients. Chin Med J (Engl) 116: 1737-1740, 2003

10. Moroni G and Ponticelli C: Rapidly progressive crescentic glomerulonephritis: Early treatment is a must. Autoimmun Rev 13: 723-729, 2014.

11. Chen A, Lee K, Guan T, He JC and Schlondorff D: Role of CD8+ T cells in crescentic glomerulonephritis. Nephrol Dial Transplant: pii: gfz043, 2019 doi: 10.1093/ndt/gfz043 (Epub ahead of print).

12. McAdoo SP and Pusey CD: Anti-glomerular basement membrane disease. Clin J Am Soc Nephrol 13: 1162-1172, 2017.

13. Kitching AR and Hutton HL: The players: Cells involved in glomerular disease. Clin J Am Soc Nephrol 11: 1664-1674, 2016

14. Rovin BH, Caster DJ, Cattran DC, Gibson KL, Hogan JJ, Moeller MJ, Roccatello D, Cheung M, Wheeler DC, Winkelmayer WC, et al: Management and treatment of glomerular diseases (part 2): Conclusions from a Kidney Disease: Improving Global Outcomes (KDIGO) controversies conference. Kidney Int 95: 281-295, 2019.

15. Lin W, Chen M, Cui Z and Zhao MH: The immunopathological spectrum of crescentic glomerulonephritis: A survey of 106 patients in a single Chinese center. Nephron Clin Pract 116: c65-c74, 2010.

16. Xiao C, Xiao P, Li X, Li X, Li H, Chen Y, Wang Y, Xu Y, Huang $G$ and Zhou Q: Cordyceps sinensis may inhibit Th22 cell chemotaxis to improve kidney function in $\lg \mathrm{A}$ nephropathy. Am J Transl Res 10: 857-865, 2018.

17. Chawla LS, Bellomo R, Bihorac A, Goldstein SL, Siew ED, Bagshaw SM, Bittleman D, Cruz D, Endre Z, Fitzgerald RL, et al: Acute kidney disease and renal recovery: Consensus report of the acute disease quality initiative (ADQI) 16 workgroup. Nat Rev Nephrol 13: 241-257, 2017.
18. Watts RA and Scott DG: Epidemiology of the vasculitides. Semin Respir Crit Care Med 25: 455-464, 2004.

19. Chen M, Cui Z and Zhao MH: ANCA-associated vasculitis and anti-GBM disease: The experience in China. Nephrol Dial Transplant 25: 2062-2065, 2010.

20. Canney M, O'Hara PV, McEvoy CM, Medani S, Connaughton DM, Abdalla AA, Doyle R, Stack AG, O'Seaghdha CM, Clarkson MR, et al: Spatial and temporal clustering of anti-glomerular basement membrane disease. Clin J Am Soc Nephrol 11: 1392-1399, 2016.

21. Chen M, Cui Z and Zhao MH: ANCA-associated vasculitis and anti-GBM disease: The experience in China. Nephrol Dial Transplant 25: 2062-2065, 2010.

22. Yuan Q, Wang J, Peng Z, Zhou Q, Xiao X, Xie Y, Wang W, Huang L, Tang W, Sun D, et al: Neutrophil-to-lymphocyte ratio and incident end-stage renal disease in Chinese patients with chronic kidney disease: Results from the Chinese Cohort Study of Chronic Kidney Disease (C-STRIDE). J Transl Med 17: 86, 2019.

23. Gan L, Li X, Zhu M, Chen C, Luo H and Zhou Q: Acteoside relieves mesangial cell injury by regulating Th22 cell chemotaxis and proliferation in IgA nephropathy. Ren Fail 40: 364-370, 2018.

24. Peng Z, Wang J, Yuan Q, Xiao X, Xu H, Xie Y, Wang W, Huang L, Zhong Y, Ao X, et al: Clinical features and CKD-related quality of life in patients with CKD G3a and CKD G3b in China: Results from the Chinese Cohort Study of Chronic Kidney Disease (C-STRIDE). BMC Nephrol 18: 311, 2017.

25. Chen Y, Li H, Xiao C, Zeng X, Xiao X, Zhou Q and Xiao P: NLRC5: Potential novel non-invasive biomarker for predicting and reflecting the progression of IgA nephritis. J Transl Med 16: 317,2018

26. Gapud EJ, Seo P and Antiochos B: ANCA-associated vasculitis pathogenesis: A commentary. Curr Rheumatol Rep 19: 15, 2017.

27. Xiao H, Hu P, Falk RJ and Jennette JC: Overview of the pathogenesis of ANCA-associated vasculitis. Kidney Dis (Basel) 1: 205-215, 2016.

28. Hedger N, Stevens J, Drey N, Walker S and Roderick P: Incidence and outcome of pauci-immune rapidly progressive glomerulonephritis in Wessex, UK: A 10-year retrospective study. Nephrol Dial Transplant 15: 1593-1599, 2000.

29. Hung PH, Chiu YL, Lin WC, Chiang WC, Chen YM, Lin SL, Wu KD and Tsai TJ: Poor renal outcome of antineutrophil cytoplasmic antibody negative Pauci-immune glomerulonephritis in Taiwanese. J Formos Med Assoc 105: 804-812, 2006.

30. Yang Y, Luo M, Xiao L, Zhu XJ, Wang C, Fu X, Yuan SG, Xiao F, Liu H, Dong Z, et al: Exploration of pathological prediction of chronic kidney diseases by a novel theory of bi-directional probability. Sci Rep 6: 32151, 2016.

31. Huart A, Josse AG, Chauveau D, Korach JM, Heshmati F, Bauvin E, Cointault O, Kamar N, Ribes D, Pourrat J, et al: Outcomes of patients with Goodpasture syndrome: A nationwide cohort-based study from the French Society of Hemapheresis. J Autoimmun 73: 24-29, 2016.

32. van Daalen EE, Jennette JC, McAdoo SP, Pusey CD, Alba MA, Poulton CJ, Wolterbeek R, Nguyen TQ, Goldschmeding R, Alchi B, et al: Predicting outcome in patients with anti-GBM glomerulonephritis. Clin J Am Soc Nephrol 13: 63-72, 2018.

33. Han F, Chen L, Le J, Choong P, Xu Y, Wang H and Chen J: The clinicopathologic spectrum of rapidly progressive glomerulonephritis based on glomerular immune deposition and antineutrophil cytoplasmic antibody. Appl Immunohistochem Mol Morphol 23: 704-710, 2015.

This work is licensed under a Creative Common Attribution-NonCommercial-NoDerivatives 4.0 International (CC BY-NC-ND 4.0) License. 